\title{
Propiedades nutraceúticas del licopeno
}

\author{
Krzysztof NWaliszewski, DSc, (I) Gabriela Blasco, MSc.(I)
}

\section{Waliszewski KN, Blasco G. \\ Propiedades nutraceúticas del licopeno. Salud Publica Mex 2010;52:254-265.}

\begin{abstract}
Resumen
Las recomendaciones dietarias en los últimos años proponen el incremento del consumo de alimentos que contienen fitoquímicos, ya que éstos proveen efectos benéficos para la salud humana y juegan un papel importante en la prevención de enfermedades crónicas. El licopeno, carotenoide responsable del color rojo de los tomates, ha atraído la atención debido a sus propiedades biológicas y fisicoquímicas en la prevención de enfermedades crónicas como cáncer, enfermedades cardiovasculares y neurodegenerativas, e hipertensión, entre otras, en las cuales el estrés oxidativo es un importante factor etiológico. Los antioxidantes, incluyendo al licopeno, interactúan con las especies reactivas del oxígeno, pudiendo mitigar el efecto dañino y jugar un papel significativo en la prevención de dichas enfermedades. Este artículo presenta una revisión de algunos estudios epidemiológicos realizados en los últimos años acerca de los efectos benéficos del licopeno en la salud humana.
\end{abstract}

Palabras clave: licopeno; alimentos funcionales; enfermedad crónica; salud humana.

\author{
Waliszewski KN, Blasco G. \\ Nutraceutical properties of lycopene. \\ Salud Publica Mex 2010;52:254-265.
}

\begin{abstract}
In recent years, dietary recommendations have suggested an increase in the consumption of foods that contain phytochemicals that provide benefits to human health and play an important role in preventing chronic diseases. Lycopene -the carotenoid responsible for the red color of tomatoeshas attracted attention because of its physicochemical and biological properties in the prevention of chronic diseases in which oxidative stress is a major etiological factor, such as cancer, cardiovascular and neurodegenerative diseases, and hypertension, among others. Antioxidants, including lycopene, interact with reactive oxygen species, can mitigate their damaging effects and play a significant role in preventing these diseases.This article presents a review of some epidemiological studies published in recent years on beneficial effects of lycopene in human health.
\end{abstract}

Keywords: functional food; health; oxidative stress.
A ctualmente se ha puesto énfasis en el desarrollo de alimentos funcionales, definidos como "alimentos que ha sido demostrado que afectan benéficamente una o más funciones en humanos, más allá de efectos nutricionales adecuados, de una manera que es relevante para incrementar su estado de salud y prevenir y/o reducir el riesgo de enfermedades". ${ }^{1}$ Con base en esto, los investigadores se han concentrado en los constituyentes dietarios menores como vitaminas y microelementos, compuestos fitoquímicos (carotenoides, flavonoides, indoles, isotiocianatos), zooquímicos (ácido linolénico conjugado y ácidos grasos $(\omega-3)$, fungoquímicos y bacterioquímicos (formados durante la fermentación por la flora microbiana).

Actualmente son conocidas nuevas funciones de los micronutrientes. Se estudian tanto como alimentos con

(I) Laboratorio de Enzimología, Unidad de Investigación y Desarrollo en Alimentos, Instituto Tecnológico de Veracruz. Veracruz, México.

Fecha de recibido: 23 de abril de 2009 - Fecha de aceptado: 16 de febrero de 2010 Solicitud de sobretiros: Dr. Krzysztof NWaliszewski. Laboratorio de Enzimología, Unidad de Investigación y Desarrollo en Alimentos, Instituto Tecnológico de Veracruz. Miguel Ángel de Quevedo 2779. 91860 Veracruz,Veracruz, México.

Correo electrónico:kw@itver.edu.mx 
origen en plantas étnicas tradicionales y extractos herbales o como derivados de frutas y vegetales comunes que contienen componentes activos llamados fitoquímicos o fitonutrientes (definidos como "metabolitos secundarios de las plantas, los cuales protegen a la planta contra diversos tipos de estrés"). ${ }^{2}$ Algunas funciones de acción biológica en el organismo en las que intervienen son: evitar el estrés oxidativo, regular la función genética, realizar modulación hormonal e inmune y participar en el metabolismo carcinogénico y en la ruta metabólica mediante la inducción de enzimas.

Los carotenoides son importantes fitoquímicos que se consideran como responsables de efectos protectores de la salud de las frutas y verduras. ${ }^{3,4}$ Los carotenoides son un grupo de cerca de 600 pigmentos liposolubles responsables de los colores naturales amarillos, naranjas y rojos de las frutas y vegetales. ${ }^{5-7}$ Estos pigmentos son tetra-terpenoides, compuestos por 40 carbonos debido a que están formados por ocho unidades isoprenoides. Todos los carotenoides son derivados del licopeno, que presenta una estructura acíclica (figura 1). ${ }^{8}$

\section{Fuentes y función del licopeno}

El licopeno es un carotenoide, principal pigmento responsable de la característica coloración roja del tomate

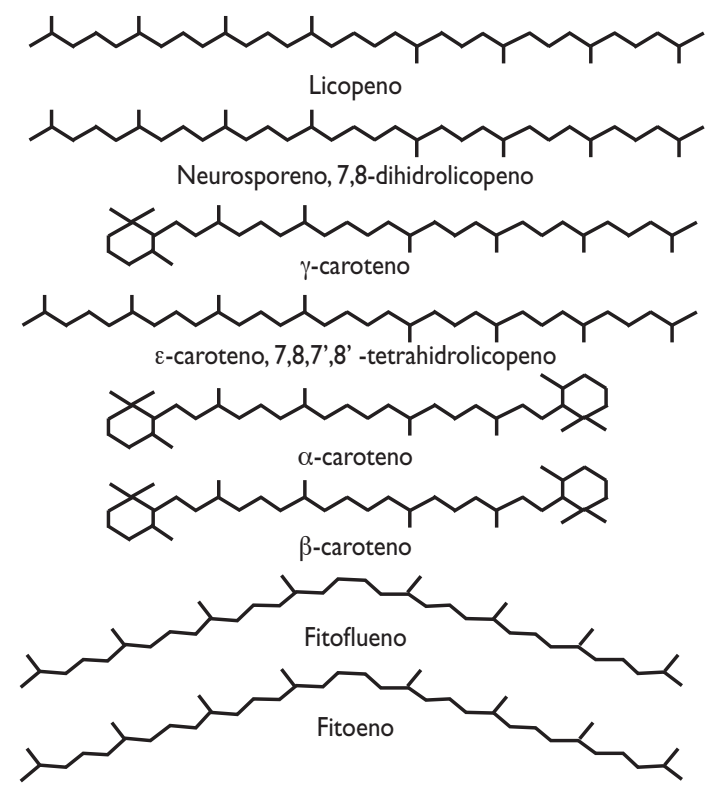

Fuente: Referencia 3

Figura I. Estructuras químicas de carotenoides en TOMATES Y PRODUCTOS A BASE DE TOMATE
(Lycopersicon esculemtum) y sus productos derivados, y se sintetiza exclusivamente por plantas y microorganismos. Una de las funciones del licopeno y otros compuestos relacionados con los carotenoides es la de absorber la luz durante la fotosíntesis, protegiendo a la planta contra la fotosensibilización. ${ }^{4}$

El tomate es un importante producto agrícola en todo el mundo. A nivel nacional, se utiliza desde antes de la conquista como alimento básico junto con el maíz, frijol y chile, ya sea crudo para ensaladas, antojitos y tortas, o como ingrediente en salsas y guisos. En otros países, a pesar de que al inicio era rechazado por su semejanza con la belladona, actualmente es un elemento insustituible, por ejemplo, en la dieta mediterránea y en muchas otras preparaciones en todo el mundo. ${ }^{9}$ Los tomates y los alimentos a base de tomate son considerados alimentos saludables por diversas razones, entre las que están presentar bajo contenido calórico y grasa, estar libres de colesterol y ser una buena fuente de fibra y proteínas. Además, los tomates son ricos en vitamina A y $C, \beta$-caroteno y licopeno. El color rojo profundo característico de los tomates sirve como parámetro de la calidad total de la fruta. Los tomates y sus productos derivados son las mayores fuentes de licopeno y son considerados importantes contribuidores de carotenoides en la dieta humana. Otras fuentes de licopeno son la sandía, la guayaba rosa, la papaya y la toronja, entre otras, como se muestra en el cuadro I. ${ }^{10-12}$

El licopeno es el carotenoide más abundante en el tomate, pues comprende aproximadamente de 80 a $90 \%$ de los pigmentos presentes. La cantidad de licopeno en tomates frescos puede variar dependiendo de la especie, la madurez y las condiciones ambientales en las que la fruta madura. ${ }^{13}$ Normalmente, los tomates contienen cerca de 3 a $5 \mathrm{mg}$ de licopeno por $100 \mathrm{~g}$ de material crudo. ${ }^{14}$ Tonucci y colaboradores reportaron que el contenido de licopeno en toda la fruta es mayor a $9.27 \mathrm{mg} / 100 \mathrm{~g} .{ }^{15}$ Algunas variedades rojas contienen más de $15 \mathrm{mg} / 100 \mathrm{~g}$, mientras que las variedades amarillas contienen sólo cerca de $0.5 \mathrm{mg} / 100 \mathrm{~g}$. Heinonen y colaboradores reportaron que la concentración de licopeno en tomates es mayor en verano (junio-agosto) y menor en invierno (octubre-marzo). ${ }^{16}$ Lampe y Watada indicaron que el contenido de licopeno puede variar de acuerdo con las técnicas de fertilización, tiempo de cosecha y variedad seleccionada. ${ }^{17}$

\section{Absorción y distribución del licopeno}

El licopeno se distribuye ampliamente en el cuerpo humano. Es uno de los mayores carotenoides que se encuentran en el suero humano (entre 21 y $43 \%$ de los carotenoides totales) con niveles en el plasma en un 
Cuadro I

\section{Contenido de licopeno en VARIOS ALIMENTOS}

\begin{tabular}{cc} 
Fuente & $\begin{array}{c}\text { Contenido de licopeno }(\mathrm{mg} / \mathrm{l00g} \text { base } \\
\text { húmeda) }\end{array}$ \\
Tomate, fresco & $0.72-20$ \\
\hline Tomate, jugo & $5.00-1 \mathrm{I} .60$ \\
\hline Tomate, salsa & 6.20 \\
\hline Tomate, pasta & 365 \\
\hline Tomate, sopa & 7.99 \\
\hline Salsa catsup & $9.90-13.44$ \\
\hline Salsa para pizza & 12.71 \\
\hline Sandía & $2.3-7.2$ \\
\hline Guayaba rosa & $5.23-5.50$ \\
\hline Toronja & $0.35-3.36$ \\
\hline Papaya & $0.11-5.3$ \\
\hline Zanahoria & $0.65-0.78$ \\
\hline Calabaza & $0.38-0.46$
\end{tabular}

Fuente: Referencia 34

rango de 0.22 a $1.06 \mathrm{nmol} / \mathrm{mL} .{ }^{18}$ Se encuentra en varios tejidos del cuerpo humano, tales como hígado, riñón, glándulas renales, testículos, ovarios y próstata. ${ }^{18,19} \mathrm{Los}$ niveles de licopeno en el plasma y tejidos humanos reflejan su ingesta dietaria. En un estudio, en el cual los sujetos consumían una dieta libre de tomate durante dos semanas, su nivel de licopeno disminuyó significativamente. ${ }^{20}$ La distribución del licopeno ocurre de la siguiente manera: una vez consumido, el licopeno es incorporado dentro de las micelas de los lípidos dietarios y absorbido en la mucosa intestinal por difusión pasiva, donde es incorporado a los quilomicrones y liberado al sistema linfático para ser transportado al hígado. El licopeno es transportado por las lipoproteínas dentro del plasma para la distribución a diferentes órganos. ${ }^{21}$ Debido a su naturaleza lipofílica, también se encuentra en las fracciones de las lipoproteínas LDL y VLDL y no en las HDL en el suero. ${ }^{5}$ El diagrama esquemático de la absorción y transportación del licopeno se muestra en la figura 2.

En humanos, la absorción de licopeno está en un rango de $10-30 \%$, con un remanente que es excretado. Algunos factores biológicos y de estilo de vida influyen en la absorción del mismo: la edad, género, estado hormonal, masa y composición corporal, niveles lipídicos en sangre, fumar, consumir alcohol y la presencia de otros carotenoides en los alimentos. ${ }^{4,5}$

\section{Mecanismo de acción biológica del licopeno}

A diferencia de otros carotenoides como el $\alpha-y$ $\beta$-caroteno, el licopeno no tiene actividad de pro vitamina A debido a que carece de la estructura de anillo $\gamma$-iónico común en estos carotenoides. ${ }^{22}$ Los efectos biológicos del licopeno en humanos se atribuyen a otros mecanismos de la vitamina A. Se han propuesto dos hi-

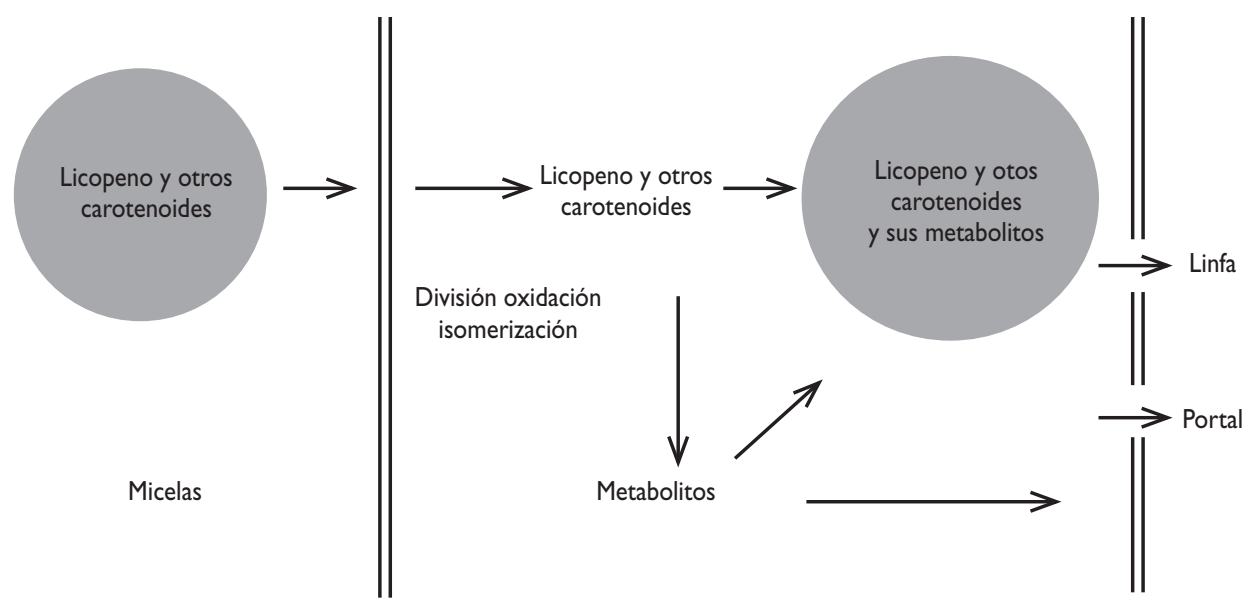

$\begin{array}{lll}\text { Mucosa } & \text { Serosal } & \text { Quilomicrón }\end{array}$

Fuente: Referencia 34

Figura 2. Absorción y tRANSPORTE del LICOPENo 
pótesis para explicar las actividades anticarcinogénicas y antiaterogénicas del licopeno: mecanismo oxidativo y no oxidativo. El mecanismo propuesto del papel del licopeno en la prevención de estas enfermedades crónicas es el que se presenta en el esquema de la figura 3.

Algunos autores estudiaron el mecanismo de acción del licopeno. ${ }^{22,23}$ La ingesta de licopeno ha mostrado un incremento en los niveles y tejidos circulatorios. Al actuar como un antioxidante, puede atrapar especies reactivas de oxígeno (EROS) y reducir el estrés oxidativo y el peligro de oxidación de los componentes celulares, incluyendo lípidos, proteínas y ADN. ${ }^{18}$ Mientras el daño oxidativo de lípidos, proteínas y ADN está implicado en el desarrollo de las enfermedades crónicas, tales como las cardiovasculares, el cáncer y la osteoporosis, el licopeno actúa como potente antioxidante que puede reducir el riesgo de padecer estos padecimientos. ${ }^{23}$

El estrés oxidativo es uno de los principales factores etiológicos de la generación de las enfermedades crónicas que afectan al ser humano. Es provocado por la alta producción de especies reactivas de oxigeno (EROS) que causan daño oxidativo en importantes biomoléculas como los lípidos, oxidando los ácidos grasos insaturados, proteínas, aminoácidos y ADN, lo que provoca daños en su estructura que, de no ser reparados, se acumulan en las células y se incrementa el riesgo de que se presente una enfermedad crónica. ${ }^{24}$ Las EROS se producen endógenamente como producto del proceso metabólico normal, o de factores de la vida diaria como la dieta, el humo de cigarro y el ejercicio. Los antioxidantes, en virtud de su habilidad de interactuar con las EROS, pueden mitigar este daño, por lo que juegan un papel importante en la prevención de las enfermedades crónicas.

\section{Cáncer y licopeno}

En México el cáncer ocupa el tercer lugar de las causas de muerte con 18\%; el más común en hombres es el de próstata, seguido del de intestino, estómago y colorrec-

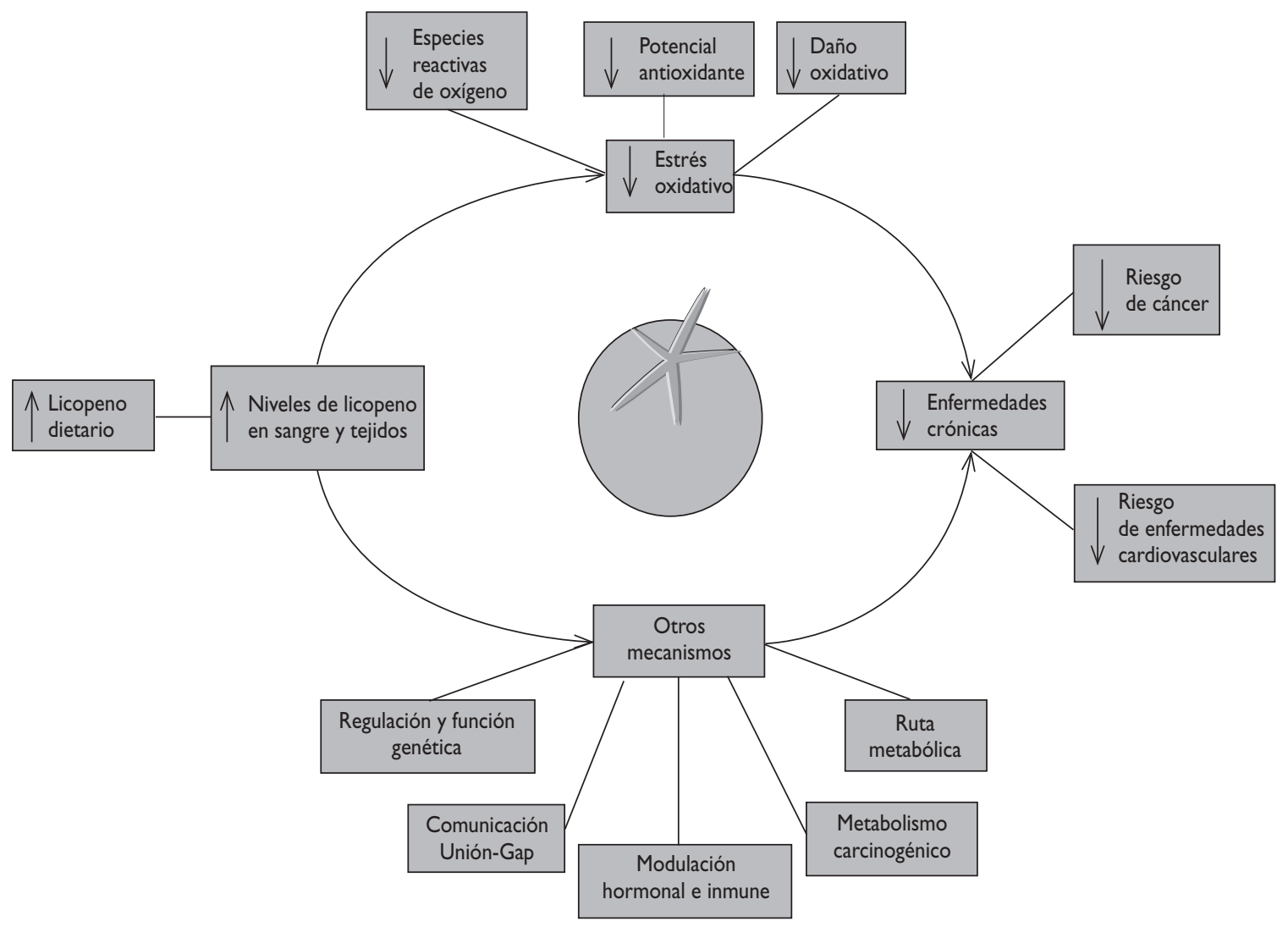

Fuente: Referencia 18

\section{Figura 3. Mecanismo propuesto para el papel del licopeno en las enfermedades CRónicas}


tal; el de cérvix es el más común en mujeres, seguido por el de seno, estómago y ovario. ${ }^{25}$ Se ha publicado un gran número de datos epidemiológicos sobre la relación entre el riesgo de padecer cáncer y la ingesta dietaria de licopeno de tomate y productos de tomate.

Con evidencia epidemiológica del posible efecto protector del licopeno contra el cáncer, diversos estudios de cultivos de células y tejidos animales han demostrado el potencial anticancerígeno del licopeno. En un estudio de cultivo celular se investigaron las propiedades de antiproliferación del licopeno en comparación con $\alpha$ - y $\beta$-caroteno, empleando células cancerosas de endometrio, glándula mamaria y de pulmón. ${ }^{26}$ Los resultados muestran que el licopeno inhibió el crecimiento de las células cancerosas en endometrio, mama y pulmón, y que el $\alpha$ - y $\beta$-caroteno fueron menos efectivos como inhibidores de crecimiento de cáncer que el licopeno. Además de este efecto inhibitorio sobre las células cancerosas de endometrio, el licopeno también suprimió el factor de crecimiento de tipo insulina-I. Los factores de crecimiento tipo insulina son los mayores reguladores autocrinos / paracrinos del crecimiento de células cancerosas en endometrio y de mama; en consecuencia, comprender el rol del licopeno al interferir en este mayor sistema autocrino/ paracrino puede contribuir al entendimiento del mecanismo por el cual el licopeno inhibe el cáncer endometrial y otros tipos de tumores. ${ }^{26}$

En un trabajo se estudió el efecto de la ingesta crónica de licopeno sobre el crecimiento y desarrollo de tumores mamarios en ratones vírgenes SHN (ratones genéticamente susceptibles al desarrollo de tumores de mama). ${ }^{26}$ Los autores encontraron que los ratones alimentados con licopeno tuvieron un ataque demorado y reducción espontánea de crecimiento y desarrollo de tumores mamarios. Esta supresión se asoció con la reducida actividad de la timidilato sintetasa de la glándula mamaria, una enzima esencial requerida para la síntesis de ADN, disminución de los ácidos grasos libres séricos y prolactina, hormona conocida por estar involucrada en el desarrollo de cáncer de pecho.

En un estudio epidemiológico se encontró que la ingesta de licopeno está correlacionada con la disminución del riesgo de padecer cáncer. ${ }^{27}$ En este estudio se monitorearon los hábitos dietéticos y la incidencia de cáncer de próstata de aproximadamente 48000 hombres durante 4 años, y se obtuvo como resultado que de cerca de 46 frutas, vegetales y productos relacionados con base en su frecuencia de consumo, sólo cuatro estuvieron significativamente asociados con un riesgo bajo de cáncer de próstata: salsa de tomate, tomate crudo, salsa para pizza a base de tomate y las fresas; las tres primeras son fuentes primarias de licopeno. Los autores encontraron que los hombres que comieron 10 o más porciones por semana de productos a base de tomate, incluyendo tomate, salsa de tomate y salsa para pizza, presentaron una disminución de $34 \%$ en la posibilidad de desarrollar cáncer de próstata, mientras que los hombres que comieron 4-7 porciones por semana sólo disminuyeron 20 por ciento. La salsa de tomate fue el predictor más fuerte en la reducción del cáncer de próstata $(66 \%)$, y el mejor predictor de niveles séricos de licopeno. ${ }^{25}$ Esta relación entre el licopeno ingerido y el riesgo bajo de cáncer de próstata fue independiente de otros factores y mayor para otros casos avanzados de cáncer. La ingesta de carotenoides tales como el $\alpha$-caroteno, $\beta$-caroteno, luteína y $\delta$-criptoxantina no estuvo asociada con la reducción del riesgo de cáncer de próstata. Los autores concluyen con estos resultados que los productos a base de tomate pueden ser especialmente benéficos en la reducción del cáncer de próstata. ${ }^{27}$

Existe una evidencia importante entre la asociación de la ingesta de licopeno y el bajo riesgo de cáncer de próstata en un estudio de salud física donde se prediagnosticaron niveles de licopeno plasmático en casi 578 casos que fueron comparados con 1294 controles. Los hombres con mayores niveles de licopeno en plasma tuvieron una reducción de aproximadamente 25\% de cáncer de próstata, y una reducción estadística significativa de $44 \%$ en el riesgo de cáncer agresivo de próstata. ${ }^{28}$ También se ha encontrado evidencia de la relación entre el licopeno y la prevención del cáncer de próstata en un estudio donde han sido examinados los niveles plasmáticos de licopeno en humanos. ${ }^{29}$

El licopeno puede también ser usado como agente terapéutico en el cáncer de próstata. Esto fue demostrado en un estudio clínico al azar para evaluar el efecto de la suplementación de licopeno en pacientes con cáncer de próstata. ${ }^{30}$ Los pacientes recibieron $15 \mathrm{mg}$ de licopeno dos veces diarias por tres semanas antes de una prostatectomía radical. Después de tres semanas de suplementación, 80\% del grupo suplementado tuvo tumores de menor tamaño en comparación con el grupo control (45\% de los sujetos) que no recibió suplementación. Estos resultados muestran que los niveles plasmáticos de antígenos de próstata específicos disminuyeron $18 \%$ en el grupo suplementado, mientras se incrementó en $14 \%$ en el grupo control. Los resultados indican que la suplementación de $30 \mathrm{mg}$ de licopeno diario puede ser suficiente para modular los marcadores clínicos de cáncer de próstata y es suficiente en el tratamiento de esta enfermedad. Sin embargo, son necesarios estudios clínicos más profundos para evaluar el potencial del licopeno como un agente terapéutico en los tratamientos de cáncer.

En los últimos años, la Fundación Mundial de Investigación sobre el Cáncer y el Instituto Americano 
de Investigación sobre el Cáncer hicieron una amplia revisión de las investigaciones realizadas sobre el efecto del licopeno en el cáncer de próstata, entre las que se encuentran cinco estudios de cohorte y nueve de casos y controles en relación con el consumo de tomate. De los estudios de cohorte, tres muestran una disminución en el riesgo de padecer esta enfermedad en los grupos que tienen una mayor ingesta de tomate, en comparación con los que tienen una ingesta menor; existe una diferencia significativa en dos estudios. El otro estudio no mostró significancia en el aumento del riesgo y un estudio reporta que no existe asociación estadísticamente significativa. Fue posible realizar un metanálisis sobre cuatro estudios de cohorte, donde se obtuvo, en resumen, un efecto estimado de 0.69 (95\% IC 0.43-1.08) por ración/ día, con moderada heterogenicidad. Uno de estos estudios reportó un efecto estimado de 0.24 (95\% IC 0.13-0.47) por $15 \mathrm{~g} /$ día por ingesta acumulada de salsa de tomate. Dos de los estudios de cohorte informaron sobre el avance o agresividad de este tipo de cáncer. Uno de estos estudios señala un riesgo estimado de 0.11 (95\% IC 0.02 - 0.70) por incremento de ración/ día para salsa de tomate y otro encontró que no había asociación estadística significativa. También fueron analizados siete estudios de casos y controles que mostraron una disminución en el riesgo de padecer cáncer de próstata en los grupos con mayor ingesta al ser comparados con los que consumían menos, donde sólo uno fue estadísticamente significativo. Un estudio indica que no existe un incremento significativo en el riesgo y otro declaró sin más detalles que no hay asociación significativa. Fue posible un metaanálisis de cinco estudios considerados relativamente de alta calidad y dos relativamente de baja calidad. El primero dio como conclusión de efecto estimado 0.97 (95\% IC 0.91 - 1.03) por ración/ día, sin heterogenicidad; el último dio, en resumen, un efecto estimado de 0.33 (95\% IC 0.04 -274) por ración/ día, con alta heterogenicidad. ${ }^{25}$

En cuanto al licopeno dietario, se analizaron tres estudios de cohorte y 14 estudios de casos y controles, en los que se encontró que dos estudios de cohorte no mostraron significancia al disminuir el riesgo de padecer cáncer de próstata en los grupos con mayor ingesta cuando se comparó con los de menor ingesta; el otro estudio tampoco mostró significancia con el aumento de riesgo de padecer la enfermedad. Fue posible realizar un metaanálisis sobre estos tres estudios antes mencionados, y resultó, en resumen, un efecto estimado de 0.97 (95\% IC 0.64-1.45) para $5 \mathrm{mg} /$ día, con baja herogenicidad. Uno de estos estudios también señala medidas acumuladas del consumo de licopeno, las cuales son una medida robusta, como el consumo a largo plazo. El efecto estimado fue de 0.95 (95\% IC 0.92-0.99) para 5 $\mathrm{mg} /$ día. Todos los estudios estuvieron completamente ajustados. Dos estudios de cohorte reportaron separadamente acerca de la enfermedad avanzada o agresiva al dar estimaciones de 0.89 (95\% IC 0.28 - 2.84) para 5 $\mathrm{mg} /$ día y 0.57 (95\% IC 0.37-0.87) para los grupos con mayor ingesta cuando se compararon con los de menor ingesta. Nueve estudios de casos y controles mostraron disminución en el riesgo para los de mayor ingesta comparados con los de menor ingesta, de los cuales sólo uno fue estadísticamente significante. Cinco estudios indicaron no ser significantes. Fue posible un metaanálisis sobre tres estudios considerados relativamente de alta calidad y sobre tres de baja calidad. El primero dio como conclusión un efecto estimado de 0.995 (95\% IC 0.95-1.04) para $5 \mathrm{mg} /$ día, sin heterogenicidad, y el último dio, en resumen, un estimado de 0.56 (95\% IC $0.23-1.36$ ) para $5 \mathrm{mg} /$ día, con alta heterogenicidad. ${ }^{25}$

También se analizaron estudios en donde se evaluó el licopeno sérico o plasmático. En este rubro se examinaron seis estudios de cohorte y dos de casos y controles, y resultó que cinco estudios de cohorte no mostraron significancia en reducir el riesgo para el grupo con mayor ingesta, comparado con los de menor ingesta; el otro estudio no mostró significancia al aumentar el riesgo. Fue posible realizar un metaanálisis sobre cuatro estudios de cohorte, y se resumió un estimado de 0.96 (95\% 0.926 - 0.999) para $10 \mu \mathrm{g} / \mathrm{L}$, sin heterogenicidad. Todos los estudios de cohorte fueron completamente ajustados. Ambos casos y controles de licopeno sérico y plasmático mostraron una reducción estadísticamente significativa para los de mayor ingesta en comparación con los de menor ingesta. ${ }^{25}$

Con toda la información de la Fundación Mundial de Investigación sobre el Cáncer y el Instituto Americano de Investigación sobre el Cáncer expuesta anteriormente, se concluye que existe una amplia evidencia, en particular sobre productos de tomate, en estudios de cohorte y de casos y controles, de mecanismos verosímiles por los que el licopeno protege contra el cáncer de próstata. ${ }^{25}$

El panel de expertos también hace mención sobre la existencia de otros dos estudios de cohorte referentes a este tipo de cáncer que ya habían sido publicados, y cuya conclusión no hacía cambiar su juicio. ${ }^{31,32}$ Uno de estos estudios es el de Kirsh y colaboradores en el que se concluye que los aparentes efectos protectores del licopeno observados no son suficientemente fuertes $o$ consistentes, y no suministran completa evidencia de que el licopeno o los productos a base de tomate protejan contra este tipo de padecimiento. También se menciona que el proceso de cocinado y el consumo frecuente de grasa podrían ser elementos necesarios para obtener los beneficios de los productos de tomate; sin embargo, un 
aumento en la ingesta de licopeno y productos a base de tomate es probablemente, en sí mismo, representante de una medida preventiva subestimada para evitar el riesgo de padecer cáncer de próstata. ${ }^{31}$ Por otro lado, el estudio realizado por Stram y colaboradores evalúa la relación que existe entre la incidencia de padecer cáncer de próstata y la ingesta de frutas, vegetales y micronutrientes; encuentra que existe una mínima evidencia de algún efecto protector en la ingesta de este tipo de alimentos sobre el riesgo de padecer cáncer de próstata y, lo que es más, informa que fueron observadas con más frecuencia asociaciones positivas que efectos protectores con la ingesta de estos alimentos. ${ }^{32}$

Además de su relación con el cáncer de próstata, está aumentando la evidencia que indica que el licopeno juega un papel importante en la prevención del cáncer en otros órganos como pecho, pulmón, o de tipo gastrointestinal, cervical, de ovario, y pancreático. ${ }^{33}$ Como en el cáncer de próstata, la principal evidencia para sostener el papel del licopeno en la prevención de estos tipos de cáncer proviene de realizar estudios futuros en cultivos celulares y animales, así como estudios epidemiológicos; hasta el momento no se han reportado estudios de intervención clínica o dietaria en el país, por lo que es imperativo que se realicen investigaciones en este ámbito, pues es una causa importante de mortalidad.

\section{Enfermedades cardiovaculares y licopeno}

Las enfermedades cardiovasculares son la mayor causa de muerte en el mundo. En América Latina y el Caribe las enfermedades cardiovasculares representan 31\% del total de las defunciones. En México, este grupo de enfermedades constituye un problema de salud pública; las enfermedades del corazón constituyen la primera causa de muerte y anualmente ocurren cerca de 70000 defunciones por este motivo y 26000 por enfermedades cerebrovasculares. ${ }^{34}$

Cabe hacer hincapié en los niveles séricos de colesterol como un biomarcador para el riesgo de padecer una enfermedad cardiovascular. El estrés oxidativo inducido por las EROS es también considerado un factor importante en la etiología de diversas enfermedades crónicas, incluyendo las cardiovasculares. ${ }^{29,36,37}$ Diversos estudios indican que el consumo de antioxidantes como el licopeno contenido en los tomates y sus productos puede reducir el riesgo de enfermedades cardiovasculares.

El licopeno ha demostrado tener efecto hipocolesterolémico in vivo e in vitro. En un pequeño estudio de suplementación dietaria, a seis hombres saludables se les dio $60 \mathrm{mg} /$ día de licopeno por tres meses. Al final del periodo de tratamiento, una significante reducción de $14 \%$ en los niveles plasmáticos de colesterol LDL fue observada in vivo, sin un efecto sobre las concentraciones de colesterol HDL. ${ }^{38}$ También los autores investigaron el efecto hipocolesterolémico del licopeno en macrófagos in vitro. Incubaron las células de macrófagos con licopeno in vitro y resultó una disminución de $73 \%$ de la síntesis de colesterol. Estos resultados sugieren que el licopeno inhibe la síntesis de colesterol de los macrófagos por una inhibición celular de la HMGCoa reductasa, enzima limitante en la síntesis de colesterol in vitro.

Existe evidencia del estudio del Factor de Riesgo de Enfermedad del Corazón Isquémico de Kuopio (KIHD por sus siglas en inglés) que sugiere que el adelgazamiento de las paredes de los vasos sanguíneos y el riesgo de un infarto al miocardio se puede reducir en personas con una mayor concentración de licopeno sérica y en tejido adiposo..$^{39}$ Este resultado demuestra que la concentración de licopeno sérico puede jugar un papel importante en las etapas tempranas de la ateroesclerosis. Una pared arterial delgada es una señal temprana de ateroesclerosis, y si incrementa la delgadez de la media íntima, se ha mostrado que puede predecir eventos coronarios. Similarmente, se investigó la relación entre la concentración de licopeno plasmático y la delgadez media íntima de las paredes de la arteria carótida común en 520 hombres y mujeres de edad media entre 45 y 69 años, como parte del Estudio de Suplementación de Antioxidantes para la Prevención de Ateroesclerosis. ${ }^{40}$ Bajos niveles de licopeno en sangre estuvieron asociados con un incremento de $17.8 \%$ en CCA-IMT en hombres, mientras no tuvo diferencia significativa en mujeres. Estos resultados también sugieren que las bajas concentraciones plasmáticas de licopeno están asociadas con la temprana ateroesclerosis, evidenciadas por el incremento de CCA-IMT en hombres de mediana edad.

Otro estudio mostró una relación inversa entre los niveles de licopeno sérico y ateroesclerosis, calculado por la presencia de plaquetas calcificadas en la aorta abdominal. ${ }^{41}$ La población estudiada comprendió 108 casos de ateroesclerosis aórtica y 109 controles de 55 años o más. La asociación entre los niveles de licopeno sérico y la ateroesclerosis fue muy importante en casi todos los sujetos que eran fumadores activos. No se presentó asociación con el riesgo de calcificación aórtica para los carotenoides séricos $\alpha$ - y $\beta$-caroteno, luteína y zeaxantina. Estos resultados sugieren que el licopeno juega un papel protector en el desarrollo de la ateroesclerosis. Resultados del Estudio Europeo de Antioxidantes, Infarto al Miocardio y Cáncer de Mama también mostraron que hombres con mayores concentraciones de licopeno en una biopsia de su tejido adiposo tuvieron una reducción de riesgo de infarto al miocardio en $48 \%$, comparado con hombres con una menor concentración de licopeno en su tejido adiposo. ${ }^{42}$ 
Un incremento en la oxidación de LDL está asociado con el riesgo de padecer ateroesclerosis y enfermedades coronarias. ${ }^{43}$ Agarwal y Rao investigaron el efecto de una suplementación dietaria de licopeno sobre la oxidación de las LDL en 19 sujetos sanos. ${ }^{44}$ La suplementación se administró usando jugo de tomate, espagueti con salsa de tomate y una oleorresina de tomate por un periodo de una semana para cada sujeto. Las muestras de sangre se colectaron al final de cada tratamiento y se midieron las concentraciones de TBARS y dienos conjugados para estimar la oxidación de las LDL. Se encontró un incremento significativo en los niveles de licopeno de por lo menos el doble; la suplementación de licopeno redujo significativamente la peroxidación de los lípidos y la oxidación de las LDL. El promedio de disminución de TBARS y de LDL-enfermedades cardiovasculares en tratamiento con productos de tomate fue de 25 y $13 \%$, respectivamente. Estos resultados sugieren que existe significancia del licopeno en el decremento del riesgo de padecer una enfermedad del corazón.

El resultado de otro estudio sobre la salud de la mujer mostró que las mujeres que tenían una mayor ingesta de alimentos a base de tomate tenían una reducción de riesgo para una enfermedad cardiovascular, comparado con las mujeres con una baja ingesta de estos alimentos. ${ }^{45}$ Los resultados mostraron que las mujeres que consumían siete porciones o más de productos de tomate a la semana tenían cerca de $30 \%$ de reducción en el total de las enfermedades cardiovasculares, comparado con el grupo que consumía menos de una porción a la semana. Los investigadores también encontraron que las mujeres que consumían más de 10 porciones a la semana tenían más pronunciada la reducción en el riesgo $(65 \%)$ para alguna enfermedad cardiovascular específica como ataque o apoplejía al corazón.

Estudios epidemiológicos han mostrado una relación inversa entre los niveles séricos y el tejido de licopeno y la morbilidad y mortalidad cardiovascular. Diversos estudios han demostrado que el licopeno es un protector contra este tipo de enfermedades. La baja concentración de HDL está asociada con el incremento de enfermedades coronarias del corazón, por lo que existen investigaciones para elucidar cómo las HDL pueden proveer protección contra la ateroesclerosis. Las enfermedades clínicas han resultado relevantes para entender el rol de las HDL en la remoción del colesterol de las paredes arteriales y se han sugerido diversas estrategias para aumentar la actividad benéfica de estas lipoproteínas. En un estudio realizado en el Hospital de Helsinki, Finlandia, se evaluaron 98 voluntarios aparentemente saludables, de los cuales 50 comieron una dieta rica en tomate de aproximadamente $300 \mathrm{~g}$ diarios por un mes y 48 continuaron con su dieta regular, sin comer tomates durante ese mes. El resultado obtenido fue que una dieta rica en tomate tiene efectos benéficos en el perfil lipídico, demostrando un decremento en los niveles de colesterol-LDL y un significante incremento de $15.2 \%$ en el colesterol-HDL. ${ }^{44}$

El estrés oxidativo y la expresión de adhesión de moléculas sobre las células del endotelio vascular están en la mira como importantes causas en la patogenia de ateroesclerosis. Ciertos nutrientes y fitonutrientes que tienen propiedades antioxidantes, como el licopeno, pueden proteger las células endoteliales por la interferencia con una disfunción celular endotelial citoquinética inducida, por lo que es importante seguir realizando investigación en estos casos para llegar a comprender el mecanismo de acción del licopeno sobre estas patologías que están presentes en gran parte de la población.

\section{Osteoporosis y otros desórdenes óseos y licopeno}

El papel del licopeno en la salud de los huesos está basado en sus propiedades como potente antioxidante; son bien conocidos el papel del estrés oxidativo en la salud de los huesos y los reportes limitados de estudios del efecto del licopeno en cultivos de células de huesos.

Las especies reactivas del oxígeno(EROS) inducidas por el estrés oxidativo están asociadas con la patogénesis de la osteoporosis y la baja densidad ósea. La evidencia epidemiológica sugiere que ciertos antioxidantes, como la vitamina $\mathrm{C}, \mathrm{E}$ y $\beta$-caroteno, pueden reducir el riesgo de padecer osteoporosis, ${ }^{34}$ por lo que se ha considerado estudiar el efecto del licopeno en este tipo de casos por su alto poder antioxidante.

Se conocen muy pocos trabajos sobre el papel del estrés oxidativo en los osteoblastos. Sin embargo, los osteoblastos pueden inducir la producción intracelular de EROS, con lo cual se puede causar una disminución en la actividad de la fosfatasa alcalina, que es parcialmente inhibida por la vitamina E, y causar la muerte de la célula. Estudios sobre los efectos del licopeno sobre osteoblastos se limitan a sólo dos reportes. ${ }^{47,48} \mathrm{Kim}$ y colaboradores estudiaron el efecto de incubación de células SaOS-2 como osteoblastos 10 -6 y 10 -5 M con licopeno, o su respectivo vehículo control, sobre la proliferación de las células y mostraron que el licopeno estimula la proliferación celular. ${ }^{47}$

También se encontró que el licopeno tenía un efecto estimulador sobre la actividad ALP, un marcador de diferenciación osteoblástica en más células maduras, pero dependía del tiempo de acción si tenía efecto inhibitorio o no sobre las células SaOS-Dex más jóvenes. En otro estudio, Park y colaboradores evaluaron el efecto del licopeno sobre células MC3T3 (células osteoblásticas 
de ratón), y encontraron que el licopeno tenía efecto inhibitorio sobre la proliferación de las células. ${ }^{48} \mathrm{Ambos}$ estudios, sin embargo, señalaron que la actividad ALP era estimulada. La discrepancia en el efecto del licopeno sobre la proliferación de las células puede ser diferente en especies o condiciones experimentales diferentes y se requieren más estudios para clarificar el papel del licopeno sobre los osteoblastos.

La posmenopausia está asociada con un incremento global en volumen de marcadores de huesos. Estos marcadores predicen la pérdida del hueso y la osteoporosis en mujeres posmenopáusicas. Rao y colaboradores investigaron la correlación inversa entre el licopeno sérico y parámetros de estrés oxidativo y marcadores de volumen de hueso en mujeres posmenopáusicas que tuvieran riesgo de osteoporosis. ${ }^{49}$ Treinta y tres mujeres de entre 50 y 60 años fueron evaluadas durante siete días en un reporte de ingesta dietaria antes de hacerles pruebas de sangre. Parámetros de estrés oxidativo, capacidad antioxidante total, licopeno sérico y marcadores de volumen de hueso ALP (formación de hueso) y colágeno tipo I de N-telopéptidos ligados (NTx) (resorción de hueso) se midieron en las muestras séricas. Los resultados más relevantes son: una correlación entre el licopeno ingerido y el licopeno sérico, que el licopeno es rápidamente absorbido por el cuerpo, que hay una significante disminución en la oxidación de proteínas como un indicativo por el incremento de tioles y una disminución de los valores NTx conforme los niveles de licopeno sérico se incrementan.

Mientras hay una significante correlación positiva entre el licopeno sérico y el licopeno dietario como determinante del reporte de alimento estimado, los reportes sostienen la hipótesis de que el licopeno dietario actúa como un efectivo antioxidante y reducen el estrés oxidativo y los marcadores de volumen del hueso. Estas observaciones sugieren un importante papel del licopeno mediante sus propiedades antioxidantes al reducir el riesgo de padecer osteoporosis.

\section{Hipertensión y licopeno}

La hipertensión es uno de los mayores problemas de salud de la población. En los resultados de la Encuesta Nacional de Salud 2000, la prevalencia actual de hipertensión arterial es de 30.05\%; en el sexo masculino la prevalencia es de $34.20 \%$ y en el femenino de 26.30 por ciento. En esta encuesta el control de la hipertensión se estima en una tasa de hipertensos conocidos de $14.3 \%$, sin tratamiento $30.4 \%$ y una tasa de hipertensos controlados de 36 por ciento. Se señala, además, que sólo $29 \%$ de los hipertensos esenciales mexicanos, tratados farmacológicamente, están controlados óptimamente (presión arterial menor de 140/90 mmHg). ${ }^{35}$

Esta condición está comúnmente asociada con la estrechez de las arterias; sin embargo, la causa exacta de la hipertensión es desconocida. Existen diversos factores y condiciones a los que se puede atribuir su ocurrencia, como factores genéticos, historia familiar de hipertensión, obesidad, sedentarismo, exceso en la ingesta de sal, consumo de alcohol y cigarrillos, estrés, edad, niveles hormonales y anormalidades en el sistema nervioso y circulatorio. ${ }^{50-52}$ Las EROS generadas endógenamente pueden afectar múltiples tejidos, ya sea directamente o a través de la depleción del óxido nítrico e inclusión de contracción y disfunción endotelial vascular, remodelamiento hipertrófico en los vasos sanguíneos y miocardio, reabsorción de la sal y decremento en la filtración glomerular en el riñón, e incremento eferente de la actividad simpática del sistema nervioso central. A pesar de que son empleados varios agentes farmacéuticos en el tratamiento efectivo de la hipertensión, existe un considerable interés en el uso de componentes naturales de alimentos para su tratamiento. Los polifenoles derivados del té verde se han estudiado en este contexto y han mostrado un efectivo control para este padecimiento. Engelhard estudió con un ensayo placebo controlado el efecto del licopeno de tomate sobre la presión sanguínea..$^{53}$ Se recluyeron para el estudio 30 pacientes con hipertensión grado I de entre 40 y 65 años de edad, sin ningún requerimiento de medicación para la presión o dislipidemias. Después de dos semanas de evaluación, los pacientes se trataron por cuatro semanas con placebo y ocho semanas en periodo de tratamiento. El tratamiento consistió en la ingesta diaria de cápsulas de extracto de tomate que contenían $15 \mathrm{mg}$ de licopeno. Los resultados no mostraron cambios significativos en la presión diastólica después de ocho semanas de tratamiento pero mostraron una considerable reducción en la presión sistólica del valor inicial de $144 \mathrm{~mm} \mathrm{Hg}$ a $134 \mathrm{~mm} \mathrm{Hg}$ al final del tratamiento con licopeno.

Para disminuir la hipertensión se recomienda una dieta aprobada. Este tipo de dietoterapia está diseñada para proveer niveles deseables de fibra, potasio, magnesio y calcio; contiene diversas frutas, vegetales y algunos granos. Comparada con dietas normales, esta dieta contiene una mayor proporción de fitoquímicos dentro de los que se encuentra un alto nivel de licopeno y otros carotenoides, polifenoles, flavonoides y flavononas, entre otros componentes. Hasta el momento no está ampliamente reconocido el efecto benéfico de estos fitoquímicos en el tratamiento de la hipertensión. ${ }^{34}$ Son necesarios más estudios clínicos e investigaciones para 
poder entender mucho mejor el papel del licopeno en este padecimiento.

\section{Infertilidad masculina y licopeno}

Se ha sugerido el estrés oxidativo como un importante factor que contribuye en la infertilidad masculina. Se han detectado niveles significativos de EROS en semen por arriba de $25 \%$ de hombres infértiles, mientras que los hombres fértiles no producen niveles detectables de EROS en su semen. ${ }^{52,53}$

La identificación de un tratamiento nuevo y menos invasivo para la infertilidad masculina, como la administración de vitaminas y antioxidantes, podría tener potencialmente un gran impacto en el tratamiento para parejas infértiles. A la fecha, pocos estudios han evaluado el papel de los antioxidantes y vitaminas (principalmente como agentes únicos) en la infertilidad masculina. En general, estos estudios sugieren un efecto benéfico de la terapia con antioxidantes en el tratamiento de infertilidad masculina, pero son necesarias investigaciones adicionales. ${ }^{54}$ Ahora que se ha reconocido al licopeno como un poderoso antioxidante y a su papel preventivo en las enfermedades crónicas provocadas por el estrés oxidativo, investigadores han empezado a estudiar su capacidad como protector de un daño oxidativo del esperma, evitando así la infertilidad.

\section{Enfermedades neurodegenerativas y licopeno}

Las enfermedades neurodegenerativas son un grupo de desórdenes degenerativos del sistema nervioso que incluye al cerebro, a la médula espinal y a los nervios periféricos. Entre estos padecimientos con diversas etiologías e importancia clínica están la enfermedad de Alzheimer, Parkinson, Huntington, esclerosis amiotrófica lateral y epilepsia. ${ }^{55}$ El estrés oxidativo está empezando a ser considerado un importante factor causal y también como factor secundario en la patogénesis de estas enfermedades.

El alto contenido lipídico del sistema nervioso, la baja capacidad antioxidante y la presencia de hierro, además de su elevada actividad metabólica, lo hacen particularmente susceptible de daño oxidativo. Diversos sistemas antioxidantes han mostrado una efectiva mitigación del efecto neurotóxico de las especies reactivas de oxígeno. ${ }^{34}$ Diversos estudios in vitro han demostrado la efectividad de los antioxidantes al proteger el tejido nervioso del daño de los radicales libres. Casi todos los antioxidantes dietarios se dirigieron en la mayoría de los estudios al papel de la vitamina A, C y E junto con el $\beta$-caroteno y mostraron una prevención del daño neuronal debido al estrés oxidativo. ${ }^{56}$ Se conocen pocos estudios sobre el papel del licopeno en las enfermedades neurodegenerativas. Un estudio presentó una reducción significativa de los niveles de licopeno en pacientes con enfermedad de Parkinson y demencia vascular. ${ }^{57}$ Se ha sugerido que los antioxidantes como el licopeno pueden actuar directamente sobre las neuronas o en forma indirecta afectando los marcadores periféricos del estrés oxidativo. ${ }^{58}$ Los niveles de licopeno en este tipo de enfermedades están presentes en muy bajas concentraciones, comparado con otros tejidos. ${ }^{59} \mathrm{Sin}$ embargo, se cree que el licopeno puede cruzar la barrera sangre-cerebro y ser efectivo en reducir el daño causado por las especies reactivas del oxígeno.

\section{Otras enfermedades y licopeno}

La mayoría de los trabajos demuestran el efecto protector del licopeno, aunque casi todos se han enfocado a la prevención de las enfermedades crónicas y cáncer, principalmente de próstata; sin embargo, el licopeno puede estar involucrado en la protección contra otro tipo de enfermedades. Esto se basa en la hipótesis de que el estrés oxidativo es un importante factor etiológico que causa la mayoría de las enfermedades degenerativas.

Han sido demostradas actividades radioprotectoras del licopeno en ratones de laboratorio y se han reportado bajos niveles de licopeno sérico en mujeres y niños con el virus de inmunodeficiencia humana positivo. Los bajos niveles de licopeno sérico en estos grupos pueden ser factores de consecuencias exacerbantes en alguna falla de su sistema inmune; esto demuestra que se necesitan nuevas investigaciones en esta área. ${ }^{60,61}$ Con referencia a otros padecimientos, la comunidad científica se ha dado a la tarea de investigar más a fondo el papel que desempeña el licopeno en enfermedades de la piel, artritis reumatoide, enfermedades periodontales y procesos inflamatorios. ${ }^{34}$

\section{Ingesta recomendada}

Actualmente no se conoce una ingesta ideal de licopeno, sin embargo, resultados de estudios epidemiológicos pueden proporcionar información sustancial sobre los niveles de licopeno que se pueden utilizar, aunque debido a la variabilidad de concentración de licopeno en la diversidad de fuentes no se ha llegado a establecer una dosis per se.

Algunos investigadores no reconocen la importancia del licopeno en la salud humana debido a la falta de actividad de provitamina A, por lo que actualmente no es considerado un nutriente "esencial". Por esta razón no se ha establecido una ingesta diaria o una ingesta 
nutrimental recomendada de licopeno. Sin embargo, con el reconocimiento del papel que el licopeno desempeña en la salud humana hay un considerable interés por parte de los nutricionistas y otros profesionales de la salud que sugieren niveles de ingesta diarios basados en conocimientos científicos. ${ }^{34}$

\section{Conclusiones}

El licopeno es un carotenoide con una importante actividad antioxidante que ha recibido un considerable interés científico en los últimos años. Sin embargo, sus propiedades antioxidantes en el aspecto químico y en estudios in vitro e in vivo se conocerán en varios años; también debe continuar estudiándose su papel en la salud humana.

\section{Referencias}

I. Henson S, Masakure O, Cranfield J. The propensity for consumers to offset health risks through the use of functional foods and nutraceuticals: The case of lycopene. Food Qual Prefer 2008; 19:395-406. 2. Holst B,Williamson G. Nutrients and phytochemicals: from bioavailability to bioefficacy beyond antioxidants. Curr Opinion Biotech 2008; 19:73-82.

3. Khachick F, Carvalho, L, Bernstein SP, Muir GJ, Zhao D, Katz NB. Chemistry, distribution, and metabolism of tomato carotenoides and their impact on human health. Exp Biol Med 2002;227:845-85I.

4. Rao AV, Agarwal S. Role of lycopene as antioxidant carotenoid in the prevention of chronic diseases: a review. Nutr Res 999;19:305-323.

5. Sthal W, Sies H. Lycopene: a biologically important carotenoid for humans? Arch Biochem Biophys 1996;336: I-9.

6. Baysal T, Ersus S, Starmans DAJ. Supercritical $\mathrm{CO}_{2}$ extraction of $\beta$-carotene and lycopene from tomato paste waste. J Agric Food Chem 2000;28:5507-55II.

7. Giovannucci E. A review of epidemiologic studies of tomatoes, lycopene, and prostate cancer. Exp Biol Med 2002;227:852-859.

8. Perera CO, Mei Yen G. Functional properties of carotenoides in human health. International J Food Proper 2007; 10:201-230.

9. Pamplona R. El poder medicinal de los alimentos. Colección dimensiones de la salud física. España:Asociación Publicadora Interamericana (APIA), 2005

10. Gross J. Pigments in fruits. London:Academic Press, 1987.

II. Gross J. Pigments in fegetables. New York:Van Nordstrand Teinhold, 1991.

12. Mangels AR, Holden JM, Beecher GR, Forman MR, Lanza E.

Carotenoids in fruits and vegetables: an evaluation of analytic data. J Am Diet Assoc 1993;93:284-296.

13. Shi J. Lycopene in tomatoes: chemical and physical properties affected by food processing. Crit Rev Food Sci Nutr 2000;40:I-42.

14. Hart DJ, Scott KJ. Development and evaluation of an HLPC method for the analysis of carotenoids in foods, and the measurement of the carotenoide content of vegetables and fruits commonly consumed in the UK. Food Chem 1995;54:10I-III.

I5. Tonucci LH, Holden JM, Beecher GR, Khachik F, Davis C, Mulokozi G. Carotenoid content of thermally processed tomato-based food products. J Agric Food Chem 1995;43:579-586.
16. Heinonen MI, Ollilainen V, Linkola EK, Varo PT, Koivistoinen PE. Carotenoids in finnish foods, vegetables, fruits, and berries. J Agric Food Chem 1989;37:655-659.

17. Lampe C,Watada AE. Postharvest quality of high pigment and crimson tomato fruit. J Amer Soc Hort Sci 1971;96(4):534-535.

18. Agarwal S, Rao VA. Tomato lycopene and its role in human health and chronic diseases. Can Med Assoc J 2000;163:739-744.

19. Cohen L.A review of animal model studies of tomato carotenoides, lycopene, and cancer chemoprevention. Exper Biol Med 2002;227:864-868. 20. Rao AV,Agarwal S. Bioavaibility and in vivo antioxidant properties of lycopene from tomato products and their possible role in the prevention of cancer. Nutr Cancer 1998;31:199-203.

2I. Parker RS. Absorption, metabolism and transport of carotenoids. FASEB J 1996; |0:542-55।.

22. Heber HN, Lu Q-L. Overview of mechanisms of action of lycopene. Exper Biol Med 2002;227:920-923.

23. Wertz K, Siler U, Goralczyk R. Lycopene: Modes of action to promote prostate health.Arch Biochem Biophys 2004;430:127-134.

24. Rao AV,Ali A. Biologically active phytochemicals in human health: Lycopene. Inter J Food Proper 2007; 10:279-288.

25. World Cancer Research Fund/American Institute for Cancer Research. Food, nutrition, physical activity, and the prevention of cancer: a global perspective. Washington DC:AICR, 2007.

26. Levy J, Bosin E, Feldman B, Giat Y, Miinster A, Danilenko M, et al. Lycopene is a more potent inhibitor of human cancer cell proliferation than either $\alpha$ - or $\beta$-carotene. Nutr Cancer 1995;24:257-266. 27. Giovannucci E,Ascherio A, Rimm EB, Stampfer MJ, Colitz GA, Willett WC. Intake of carotenoides and retinol in relation to risk of prostate cancer.J Natl Cancer Inst 1994;87: 1767- 1776.

28. Chan JM, Stampfer M, Giovannucci EL. What causes prostate cancer? A brief summary of the epidemiology. Cancer Bio 1998;8:263-273.

29. Omoni AO,Aluko RE. The anti-carcinogenic and anti-atherogenic effects of lycopene: a review. Trends Food Sci Technol 2005; I 6:344-350. 30. Kucuk O, Sarkar FH, Sak, W, Djuric Z, Pollak MN, Khachik F, et al. Phase II randomized clinical trial of lycopene supplementation before radical prostatectomy. Cancer Epidemiol Biomarkers Prev 2002;10:86I-868. 3I. Kirsh VA, Mayne ST, Peters U, Chatterjee N, Leitzmann MF, Dixon BL, et al. A prospective study of lycopene and tomato product intake and risk of prostate cancer. Cancer Epidemiol Biomarkers Prev 2006; 15(I):92-98. 32. Stram DO, Hankin JH,Wilkens LR, Park S, Henderson BR, Nomura AMY, et al. Prostate cancer incidence and intake of fruits, vegetables and related micronutrients: the multiethnic cohort study (United States). Cancer Causes Control 2006; 17:1 193-I 207.

33. Giovannucci E. Tomatoes, tomato-based products, lycopene, and cancer. Review of the epidemiologic literature. J Natl Cancer Inst 1999:91:317-331.

34. Rao AV, Ray MR, Rao LG. Lycopene.Adv Food Nutr Res 2006;51:99-164. 35. Olaiz G, Rojas R, Barquera S, Shamah T, Aguilar C, Cravioto P, et al. Encuesta Nacional de Salud 2000 Tomo II[monografía en internet]. México, DF: Instituto Nacional de Salud Pública y Secretaría de Salud, 2000 [consultado 2009 enero 20]. Disponible en http://www.insp.mx/ ensa/ensa_tomo2.pdf.

36. Witztum JL. The oxidation hypothesis of atherosclerosis. Lancet 1994:344:793-796

37. Parthasarathy S, Steinberg D,Witztum JL. The role of oxidized lowdensity lipoproteins in pathogenesis of atherosclerosis. Am Rev Med 1992;43:219-225.

38. Fuhrman B, Elis A,Aviram M. Hypocholesterolemic effect of lycopene and $\beta$-carotene is related to suppression of choresterol synthesis and augmentation of LDL receptor activity in macrophages. Biochem Biophys Res Commun 1997;233:658-662. 
39. Rissanen TH, Voutilainen S, Nyyssonen K, Salonen R, Salonen JT. Low plasma lycopene concentration is associated with increased intima-media thickness of the carotid artery wall.Arteriosclero Thromb Vasc Biol 2003;20:2677-2681.

40. Rissanen TH,Voutilainen S, Salonen R, Kaplan GA, Salonen JT. Serum lycopene concentration and carotid atherosclerosis: the Kupio Ischaemic Heart Disease Risk Factor Study. Am J Clin Nutr 2000;77:133-138. 4I. Klipstein-Grobusch K, Launer LJ, Geleijnse JM, Boeing H, Hofman A, Witteman JCM. Serum carotenoids and atherosclerosis -The Rotterdam study.Atherosclerosis 2000;148:49-56.

42. Kohlmeir L, Kark JD, Gómez-García E, Martin BC, Steck SE, Kardinaal $\mathrm{AF}$, et al. Lycopene and myocardial infarction risk in the EURAMIC study. Am J Epidemiol 1997; 146:618-626.

43. Parthasarathy S. Mechanisms by which dietary antioxidants may prevent cardiovascular diseases.J Med Food 1998; 1:45-5I.

44. Agarwal A, Rao AV.Tomato lycopene and low density lipoprotein in oxidation: a human dietary intervention study. Lipids 1998;33:98I-984.

45. Sesso HD, Liu S, Gaziano MJ, Buring JE. Dietary lycopene, tomatobased food products and cardiovascular disease in women.J Nutr 2003; 133:2336-2341.

46. Blum A, Merei M, Karem A, Blum N, Ben-Arzi S,Wirsansky I, et al. Effects of tomatoes on the lipid profile. Clin Exp Med 2006;29:5,298-300. 47. Kim L, Rao AV, Rao LG. Lycopene II- Efect on osteoblasts: The carotenoide lycopene stimulates cell proliferation and alkaline photophatase activity of SaOS-2 cells. J Med Food 2003;6:79-86. 48. Park CK, Ishimi Y, Ohmura M, Yamaguchi M, Ikegami S.Vitamin A and carotenoids stimulate differentiation of mouse osteoblastic cells.J Nutr Sci Vitaminol 1997;43:28I-296.

49. Rao LG, Mackinno ES, Josse RG, Murray TM, Strauss A, Rao AV. Lycopene consumption decreases oxidative stress and bone resorption makers in postmenopausal women. Osteoporos Int 2007; 18:109-1/5.
50. Zini A, de Lamirande E, Gagnon C. Reactive oxygen species in semen of infertile patients: levels of superoxide dismutase and catalase-like activities in seminal plasma and spermatozoa. Int J Androl 1993;16:183-188.

5I. Friedman J, Peleg E, Kagan T, Shnizer S, Rosenthal T. Oxidative stress in hypertensive, diabetic, and diabetic hypertensive rats. Am J Hypertens 2003; 16:1049-1052.

52. Lassegue B, Griending KK. Reactive oxygen species in hypertension. Am J Hypertens 2004; 17:852-860.

53. Engelhard YN, Gazer B, Paran E. Natural antioxidants from tomato extract reduce blood pressure in patients with grade-I hypertension: a double-blind, placebo-controlled pilot study.Am Heart J 2006;151:100. e6-I00.el

54. Iwasaki A, Gagnon C. Formation of reactive oxygen species in spermatozoa of infertile patients. Fertil Steril 1992;57:409-416.

55. Rao AV, Balachandran B. Role of oxidative stress and antioxidants in neurodegenerative diseases. Nutr Neurosci 2003;5:291-309.

56. Mitchell J], Paiva M, Heaton MB.Vitamin E and Beta-carotene protect against ethanol combined with ischemia in an embryonic rat hippacapal culture model of fetal alcohol syndrome. Neurosci Lett 1999;263:189-192. 57. Foy CJ, Passmore AP, Vahidassr MD, Young IS, Lawson JT. Plasma chainbreaking antioxidants in Alzheimer's disease. QJM 1999;92:39-45.

58. Sinclair AJ, Bayer AJ, Johnston J, Warner C, Maxwell SR. Altered plasma antioxidants status in subjects with Alzheimer's disease and vascular dementia. Int J Geriatr Psychiatry 1998;13;840-845.

59. Clinton SK. Lycopene: Chemistry, biology, and implications for human health and disease. Nutr Rev 1998; 1:35-5I.

60. Cooddley GO, Codley MK, Nelson HD. Micronutrients in HIV-infected women.JWomens' Health 1995;4:303-3II.

61. Periquet BA, Jammes NM, Lambert WE, Tricoire J, Maoussa MM, Garcia J, et al. Micronutrient levels in HIV-I-infected children. AIDSI 995;9:887-893. 